\title{
The Relationship Between Cognitive Status and Known Single Nucleotide Polymorphisms in Age-Related Macular Degeneration
}

\author{
Caitlin Murphy ${ }^{1,2,3 *}$, Aaron P. Johnson ${ }^{2,3}$, Robert K. Koenekoop ${ }^{4}$, William Seiple ${ }^{5,6}$ and \\ Olga Overbury ${ }^{1,7}$ \\ 'Low Vision Lab, School of Optometry, University of Montreal, Montreal, QC, Canada, ${ }^{2}$ Concordia Vision Labs, Department \\ of Psychology, Concordia University, Montreal, QC, Canada, ${ }^{3}$ Centre for Interdisciplinary Research in Rehabilitation of \\ Greater Montreal (CRIR)/Centre de Réadaptation Lethbridge-Layton-Mackay du Centre Intégré Universitaire de Santé et de \\ Services Sociaux du Centre-Ouest-de-l'lle-de-Montréal (CIUSSS) du Centre-Ouest-de-l'île-de-Montréal, Montreal, QC, \\ Canada, ${ }^{4}$ Paediatric Surgery and Human Genetics and Ophthalmology, Faculty of Medicine, McGill University Health Centre, \\ Montreal QC, Canada, ${ }^{5}$ Arlene R. Gordon Research Institute, Lighthouse Guild, New York, NY, United States, ${ }^{6}$ School \\ of Medicine, New York University, New York, NY, United States, ' Lady Davis Institute of Medical Research, Montreal, QC, \\ Canada
}

\section{OPEN ACCESS}

Edited by:

Bamini Gopinath,

The University of Sydney, Australia

Reviewed by:

Henri Leinonen,

University of California, Irvine,

United States

Elisa Mitiko Kawamoto,

University of São Paulo, Brazil

*Correspondence:

Caitlin Murphy

caitlin.murphy@mail.concordia.ca;

Cait.murf@gmail.com

Received: 23 July 2020 Accepted: 15 September 2020 Published: 16 October 2020

Citation:

Murphy $C$, Johnson AP, Koenekoop RK, Seiple W and Overbury O (2020) The Relationship Between Cognitive Status and Known Single Nucleotide Polymorphisms in Age-Related Macular Degeneration.

Front. Aging Neurosci. 12:586691.

doi: 10.3389/fnagi.2020.586691
Recent literature has reported a higher occurrence of cognitive impairment among individuals with Age-related Macular Degeneration (AMD) compared to older adults with normal vision. This pilot study explored potential links between single nucleotide polymorphisms (SNPs) in AMD and cognitive status. Individuals with AMD $(N=21)$ and controls $(N=18)$ were genotyped for the SNPs CFHY402H, ARMS2A69S and FADS1 rs174547. Cognitive status was evaluated using the Montreal Cognitive Assessment. The two groups differed significantly on which subscales were most difficult. The control group had difficulty with delayed recall while those with AMD had difficulty on delayed recall in addition to abstraction and orientation. Homozygous carriers of the FADS1 rs174547 SNP had significantly lower scores than heterozygotes or noncarriers on the MoCA. The results suggest that the FADS1 SNP may play a role in visual impairment/cognitive impairment comorbidity as reflected in the poorer cognitive scores among homozygotes with AMD compared to those carrying only one, or no copies of the SNP.

Keywords: age-related macular degeneration, mild cognitive impairment, low vision, genetics, complement factor $\mathrm{H}$, age-related maculopathy susceptibility gene 2 , fatty acid desaturase 1

\section{INTRODUCTION}

With the aging of the population, the number of individuals affected by Age-related Macular Degeneration (AMD) is on the rise. AMD is presently the leading cause of legal blindness in industrialized nations, with a prevalence that increases with age (Klein et al., 2007). To date, AMD is understood to be a retinal degenerative condition that impairs, among others, the ability to read, to recognize faces, and to drive, all of which can lead to a decreased quality of life and loss of autonomy.

In addition to a higher prevalence of AMD, older adults $(60+$ years old $)$ have a higher prevalence of cognitive impairment. Cognitive impairment refers to a decrease in a person's ability to remember and think, to an extent that it interferes with the ability to perform daily activities. At present, the World Health Organization estimates that $\sim 5-7 \%$ of the population aged 60 and 
over have some type of cognitive impairment, with the most common type being Alzheimer's disease (AD) (World Health Organization, 2012; Wortmann, 2012). There is a growing body of scientific literature linking both AMD and cognitive impairment. At the turn of the millennium, large-scale population-based studies began reporting a higher prevalence of cognitive impairment among individuals with AMD (Klaver et al., 1999; Wong et al., 2002). In the first decade of the 2000s, researchers determined the shared risk factors and histopathological characteristics (Terai et al., 2001; Johnson et al., 2002; Anderson et al., 2004; Katta et al., 2009). Most notably, beta-amyloid $(\beta \mathrm{A})$, best known as a component of the senile plaques found in the brains of individuals with $\mathrm{AD}$ was also identified as a component of drusen, the hallmark deposits of AMD (Johnson et al., 2002). Further, beta-amyloid was found to form similar vesicular structures in senile plaques and drusen (Anderson et al., 2004).

With further advances in science and technology, and based upon the data released from genome-wide association studies (GWAS), researchers have refined their abilities to study complex diseases, i.e., diseases caused by a combination of genetic, environmental and lifestyle factors. Characterizing the contribution of a single factor to a complex disease is difficult due to it being obscured or confounded by other contributing factors (Craig, 2008). A reasonable place to start is the examination of the relationship between genetic factors and associated phenotype. Such associations can lead to better knowledge of disease mechanisms and to treatment options. $\mathrm{AMD}$ and $\mathrm{AD}$ are prime examples of complex diseases. Both have benefited from the information gained from GWAS but have, so far, been studied separately in terms of genetic factors and associated phenotypes.

The current pilot study investigated AMD-cognitive impairment comorbidities with respect to possible common genetic factors. Similar disease risk factors in AMD and AD and common histopathology lead to the hypothesis that gene mutations may result in common pathogenesis in AMD and AD. The first mutation to be associated with AMD was the $\mathrm{Y} 402 \mathrm{H}$ single nucleotide polymorphism (SNP) in Complement Factor $\mathrm{H}(\mathrm{CFH})$. $\mathrm{CFH}$ is the gatekeeper for the complement cascade, and a mutation impairing its function results in increased inflammation. This association was reported by four studies in 2005 (Edwards et al., 2005; Hageman et al., 2005; Haines et al., 2005; Klein et al., 2005). Inflammation was first associated with $\mathrm{AD}$ in 1907 by Aloysius Alzheimer himself (Alzheimer et al., 1995). In addition, $\beta A$, a feature of both AMD and AD has been shown to trigger the complement cascade (Johnson et al., 2002). Considering that complement-driven inflammation and $\beta \mathrm{A}$ are implicated in both $\mathrm{AMD}$ and $\mathrm{AD}$, the same polymorphisms that infer risk for AMD may also modulate AD risk.

A second SNP having a significant impact on AMD risk is Age-related Maculopathy Susceptibility gene 2 (AMRS2) A69S. Compared to CFH, ARMS2 is not as well characterized. Research to date has found that it is expressed in both the brain and in the retina (Gatta et al., 2008). A recent study finding that ARMS2 may be involved in complement-mediated clearance of cellular debris (Micklisch et al., 2017). The A69S SNP appears to cause mRNA instability, resulting in a deficiency of the protein.
Without the ARMS2 protein present, the complement cascade is not activated to clean up necrotic cells and unwanted debris. This can lead to the formation of drusen and senile plaques that are the characteristics observed in AMD and AD respectively.

The Fatty Acid Desaturase 1 (FADS1) SNP, rs174547, has also been identified as a contributing factor toward AMD through GWAS (Neale et al., 2010), but its role is even less well characterized. The FADS1 gene encodes an enzyme involved in lipid metabolism, one of the pathogenic systems contributing to $\mathrm{AMD}$ and $\mathrm{AD}$. More specifically, FADS1 encodes delta5 fatty acid desaturase, the rate-limiting enzyme required for polyunsaturated fatty acid (PUFA) biosynthesis. It is involved in the omega-3 and omega-6 pathways (Martinelli et al., 2008; Dumont et al., 2011). The rs174547 SNP causes an increase in enzyme activity, which is thought to contribute to AMD in two ways. First, omega- 3 and omega- 6 pathways compete for use of the delta- 5 enzyme, with the omega- 6 pathway coming out ahead. This means there is always a lower level of omega3 PUFAs compared to omega-6. Due to this, there is less docosahexaenoic acid (DHA), a long-chain omega-3 fatty acid that accounts for $50 \%$ of the lipid content of photoreceptor rod outer segments (Cakiner-Egilmez, 2008; Augood et al., 2008; Tuo et al., 2009). Retinal function depends on DHA and deficiencies of omega-3 PUFAs have been shown to alter photoreceptor function (Cakiner-Egilmez, 2008). Secondly, omega-6 PUFAs compete with omega-3s for incorporation into cell membranes. The presence of omega-3s in cell membranes serves to dampen inflammatory response but, without them, the inflammation brought about by high levels of pro-inflammatory omega-6 PUFAs can go unchecked (Cakiner-Egilmez, 2008; Serini et al., 2011).

In terms of $\mathrm{AD}$, the condition of the FADS1 gene product is important for the structural integrity of the brain. Approximately half of the brain's dry mass is composed of omega-3 PUFAs, the lipids that depend on FADS1 for their biosynthesis, and $\sim 90 \%$ of this is DHA (Weiser et al., 2016). DHA is used in the phospholipid membranes of brain cells and also serves as a precursor for bioactive molecules required for brain function (Freemantle et al., 2012). It is enriched at synaptic terminals and changes in its concentration can affect cellular characteristics and physiological processes such as neurotransmitter release, signal transduction, neuroinflammation and neuronal differentiation and growth (Uauy and Dangour, 2006; Orr and Bazinet, 2008).

The current pilot study examined the frequency of the SNPs CFHY402H, ARMS2A69S, and FADS1 rs174547, and cognitive status in individuals with AMD and controls. The SNPs were expected to occur more frequently in the AMD group compared to controls and in individuals scoring positive for mild cognitive impairment. Cognitive status was measured using the Montreal Cognitive Assessment (MoCA), which has been shown to be sensitive to mild cases of cognitive impairment (Dag et al., 2014).

\section{MATERIALS AND METHODS}

Participants were recruited from the Montreal Retina Institute and the School of Optometry Clinic at the Université de 
Montréal. The study protocol was approved by Le Comité d'éthique de la recherche en santé at the Université de Montréal, and followed the tenets of the Declaration of Helsinki. All study participants gave signed informed consent prior to their participation in the study.

Individuals aged 70 years or older and diagnosed with AMD by an ophthalmologist or optometrist were recruited for this study. The control group consisted of participants aged 70 years or older recruited through word of mouth. They were required to have normal vision as determined by Early Treatment of Diabetic Retinopathy visual acuity and healthy retinas as determined by optical coherence tomography. Those with comorbid glaucoma, neurological disorders, or a diagnosis of dementia were excluded.

For the 107 participants from the Montreal Retina Institute, genotyping was conducted as part of a previous study (Smailhodzic et al., 2012a) by Radboud University Medical Center in Nijmegen, Netherlands. The remaining patients and all control participants were genotyped through Sanger sequencing-based targeted mutation analysis by Asper Biogene Ltd. In Estonia. DNA was extracted from participate saliva samples. Presence of the Y402H SNP in CFH (rs1061170), the A69S SNP in ARMS2 (rs104909245), and rs174547 SNP in FADS1 was reported.

\section{COGNITIVE ASSESSMENT}

The MoCA is designed to detect mild cognitive impairment. It screens several cognitive domains for a total score of 30 points. Individuals scoring less than 26 points are considered to have screened positive for mild cognitive impairment (Nasreddine et al., 2005). A blind version has been validated for use in visually impaired individuals (Wittich et al., 2010; Jefferis et al., 2012). The MoCA Blind omits questions requiring vision (e.g., copying a shape) and recalculates the total. It is scored out of 22, and considers a score below 18 points as screening positive for mild cognitive impairment. However, the removal of the visual components of these questionnaires reduces the overall sensitivity of the MoCA (Busse et al., 2002), leading to underestimation of scores. It is recommended to use the full version when possible, and interpret the score for all questions (full MoCA) and only the non-visual questions (MoCA Blind) separately (Busse et al., 2002).

\section{ANALYSIS}

Since the data were not normally distributed with a skewness of $-0.810(S E=0.378)$ and kurtosis of $0.310(S E=0.741)$, the non-parametric Mann-Whitney $U$-test was used to compare ranks of the cognitive measures between and within the AMD group and the control group. The same test was also used to compare data between carriers and non-carriers of each SNP of interest. A Kruskal-Wallis non-parametric one-way ANOVA was used to compare the results of cognitive tests across zygosity for each SNP. To provide more information about the distribution of these data (which can be more informative than differences between mean/median; Rousselet et al., 2017), data were divided into deciles with $95 \%$ confidence intervals. The differences between deciles in the AMD vs. Control groups were calculated to identify any meaningful difference between groups. All calculations were conducted using SPSS software, version 20.0. and JASP version 0.8.1.2 (IBM Corporation, 2011; JASP Team T, 2017).

\section{RESULTS}

A total of 107 individuals were genotyped from a previous study (Smailhodzic et al., 2012b). From this sample, mortality or development of $\mathrm{AD}$ since genotyping excluded 15 potential participants, six were unreachable, two were excluded because they were under 70 years of age, and 74 declined further participation. This left a sample of 10 individuals (3M, 7F) who completed the test battery. An additional 11 participants were added to the AMD group for a total of 21 individuals $(4 \mathrm{M}, 17 \mathrm{~F})$ with an average age of 78.9 years (range: 71-92) and average binocular visual acuity of $0.27 \log$ MAR (range: $-0.10-1.00$ $\log \mathrm{MAR})$. The control group consisted of 18 individuals $(6 \mathrm{M}$, $12 \mathrm{~F}$ ) with an average age of 74.1 years (range: $70-85$ ) and average visual acuity of $-0.02 \log$ MAR (range: $-0.26-0.16 \log$ MAR).

Genetic testing determined that there were 21 carriers of CFHY402H, with $50 \%$ of AMD and $9 \%$ of control participants being homozygous. There were also 21 carriers of ARMS2A69S, with $41.6 \%$ of AMD and $11 \%$ of control participants being homozygous. There were 33 carriers of the FADS1 SNP with 53\% of AMD and $37.5 \%$ of control participants being homozygous. The results of genetic testing are summarized in Table $\mathbf{1 .}$

\section{COGNITIVE QUESTIONNAIRES}

The MoCA scores were not significantly different between groups $($ AMD median $=27$; Control median $=28.5), U=150.00$, $p=0.27$ (Figure 1). Seven AMD participants (33.3\%) and five control participants (27.8\%) screened positive for mild cognitive impairment (MCI) according to the MoCA. When scores for the blind version of the MoCA were calculated, only one of the AMD subjects who screened positive for MCI in the original scoring achieved a score inside the normal range ( $\geq 18$ out of 22 ).

Although the average MoCA scores did not differ between the AMD group and controls, the subscales they had difficulty with did. Those from the control group scoring positive for

TABLE 1 | Genetic results.

\begin{tabular}{lccc}
\hline SNP & AMD & Control & Total \\
\hline CFHY402H (rs1061170) & 10 & 11 & 21 \\
Homozygotes & 5 & 2 & 6 \\
ARMS2A69S (rs10490924) & 12 & 9 & 21 \\
Homozygotes & 5 & 1 & 6 \\
FADS1 (rs174547) & 17 & 16 & 33 \\
Homozygotes & 9 & 6 & 15
\end{tabular}




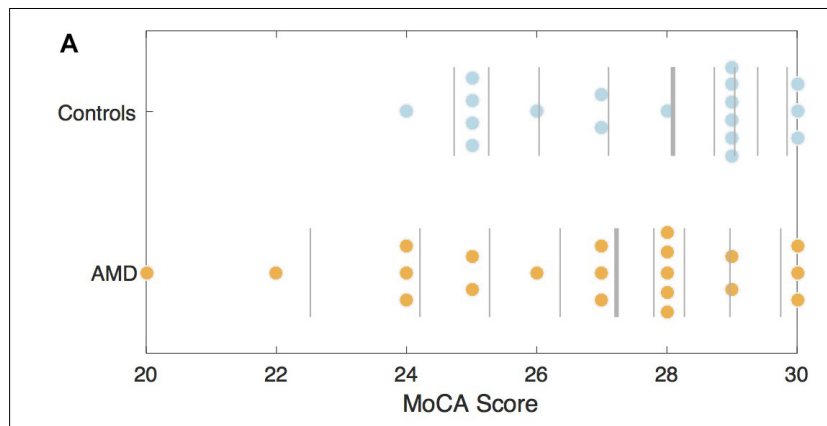

B

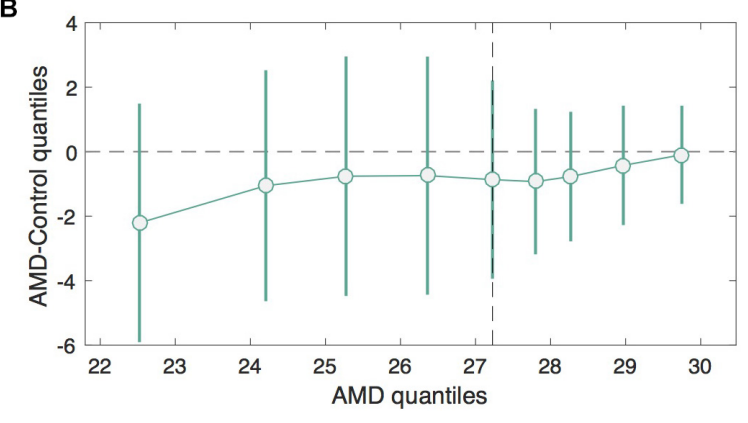

FIGURE 1 | MoCA Scores in the AMD group v. Controls. (A) Jittered scatterplots of the MoCA scores for the Control group (blue) and the AMD group (orange). The vertical lines denote deciles for each group. The thickest vertical line in each group represents the median. (B) The shift function or differences in corresponding deciles between control and AMD groups with 95\% bootstrapping confidence intervals. The deciles for the control group are plotted on the $x$-axis and the differences between control and AMD deciles are plotted on the $y$-axis. The difference is greatest for the first decile, since AMD participants had lower scores, but the confidence intervals cross zero, indicating this difference is not significant.

MCI had significantly lower scores on the delayed recall subscale compared to those from the same group who passed, $U=2.5, p=0.002$. Comparatively, those from the AMD group with MCI scored significantly poorer on delayed recall, $U=14.5, p=0.005$, in addition to the orientation, $U=37.5$, $p=0.034$, and abstraction, $U=24.5, p=0.007$, subscales of the MoCA compared to the rest of the AMD group. See Supplementary Material for details of the results of the cognitive questionnaires.

\section{GENETIC TESTING}

The CFHY402H SNP was carried by seven of the 12 who scored below normal on the MoCA. Two of them were homozygotes, neither of which had AMD. Scores obtained by carriers and non-carriers of $\mathrm{CFHY} 402 \mathrm{H}$ were not significantly different. There was no relationship between $\mathrm{CFH}$ zygosity and cognitive scores.

The ARMS2 SNP was carried by five of the individuals who scored below the normal range on the MoCA. They were all heterozygous for the SNP, with four of them being from the AMD group and one from the control group. MoCA scores did not differ significantly between carriers and non-carriers of ARMS2A69S. There was no relationship between ARMS2 zygosity and cognitive scores.

All 12 of those scoring positive for cognitive impairment on the MoCA were carriers of the FADS1 SNP. Six of the seven from the AMD group with MCI were homozygotes, while three of the five from the control group were homozygotes. The proportion of homozygotes did not differ significantly between groups, $\chi^{2}(2)=1.26, p=0.53$. Kruskal-Wallis showed that homozygous carriers of the FADS1 SNP had lower cognitive scores compared to heterozygous carriers and non-carriers, $H=8.52, p=0.014$, $\varepsilon^{2}=0.224$ (Figure 2). FADS1 SNP homozygotes with AMD had particular difficulty on the language and abstraction subscales.

\section{DISCUSSION}

A number of studies have reported an association between AMD and cognitive impairment (Klaver et al., 1999; Wong et al., 2002; Areds Research Group, 2006; Pham et al., 2006; Baker et al., 2009; Guo et al., 2010; Whitson et al., 2010; OhnoMatsui, 2011; Kaarniranta et al., 2011; Whitson et al., 2011; Woo et al., 2012; Proitsi et al., 2012; Jonna et al., 2013; Keenan et al., 2014; Rozzini et al., 2014; Chung et al., 2015; Demirci et al., 2015; Harrabi et al., 2015; Tsai et al., 2015; Zhou et al., 2016). However, these studies have been limited regarding the evaluation of cognitive impairment. While some studies have used subscales of neuropsychological tests, such as the Wechsler Adults Intelligence Scale to assess cognitive function (Areds Research Group, 2006), these subscales cannot be used to reach

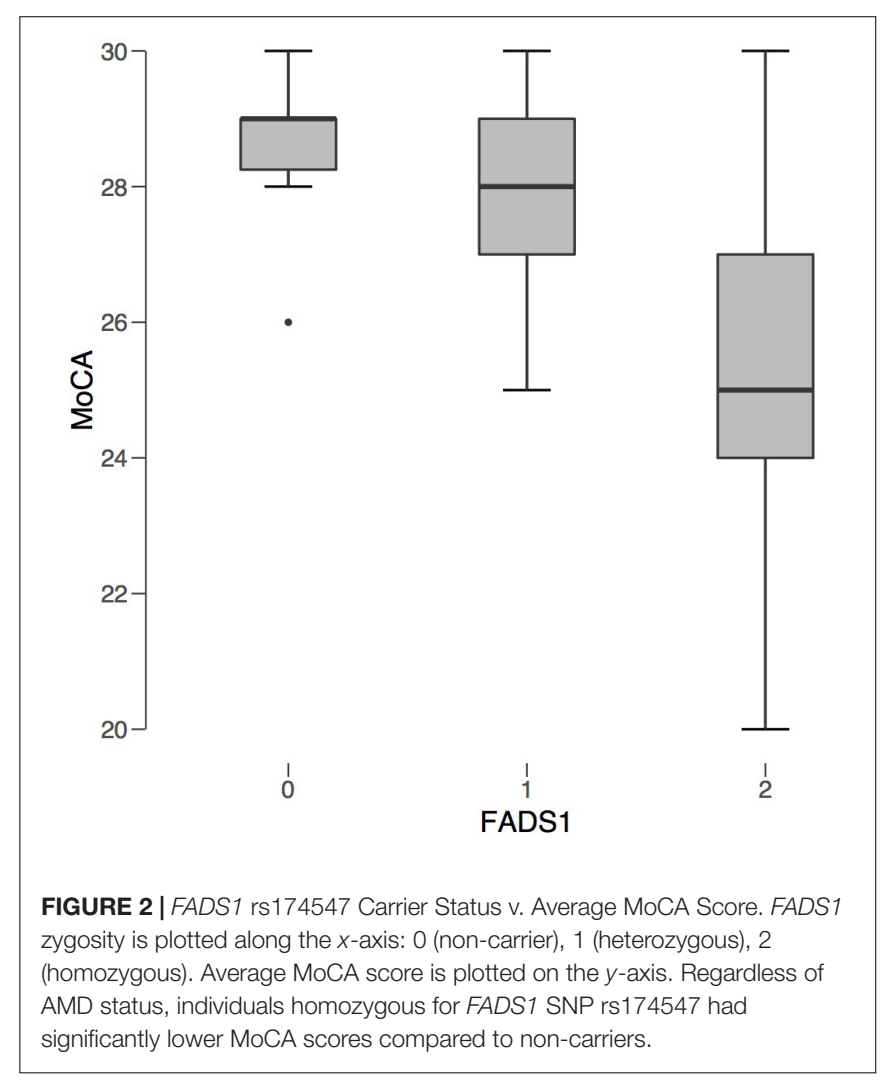


a clinical diagnosis of cognitive impairment. Other studies have used the MMSE and found an association between cognitive impairment and late AMD, but not early AMD (Baker et al., 2009). There is evidence that the MMSE is not as sensitive to MCI (Hoops et al., 2009; Dag et al., 2014). As a result, it is possible that earlier stages of AMD could be associated with milder cognitive impairment that is too subtle to be detected by the MMSE, but which could be detected by the MoCA.

The percentage of AMD participants in this study who screened positive for MCI according to the MoCA (33\%) is in agreement with previous studies (Duponsel et al., 2010; Wittich et al., 2014). The percentage of controls screening positive for MCI on the MoCA (27.8\%) was high compared to MCI prevalence reported elsewhere in older adults (Lopez et al., 2003; Gauthier et al., 2006). Four of these five control subjects scored just under the standard cut off value of 26 (on the full MoCA).

Overall full (out of 30) and blind (out of 22) MoCA scores were not significantly different between the AMD group and the control group. However, the groups did differ on which subscales of the MoCA were difficult for participants. Those in the control group who screened positive for MCI on the MoCA had difficulty with delayed recall, which is typical of an MCI diagnosis (Rozzini et al., 2007; Summers and Saunders, 2012). Not all cases of MCI progress to AD. Prospective research has shown that cases of MCI presenting with deficits in memory in addition to deficits in other cognitive domains are more likely to convert to $\mathrm{AD}$ (Rozzini et al., 2007; Summers and Saunders, 2012). Those with AMD who screened positive for MCI on the MoCA had difficulty on the delayed recall subscale, but in addition also had difficulty with the orientation and abstraction subscores. This leads to the hypothesis that those with AMD screening positive for MCI on the MoCA may be at a higher risk of developing $\mathrm{AD}$ compared to controls. Prospective studies would have to be conducted to confirm this.

A greater number of women are affected by cognitive impairment and AMD compared to men. Our results agree with this. Originally this was thought to be due to differences in life span between men and women, but recent research has shown the relationship to be more complex than that. The biological role of sex in cognitive impairment, particularly AD, and AMD is largely understudied. Recent literature has reported exaggerated aging, a faster decline from MCI to AD, and a greater effect of amyloid pathology in females compared to males (Zhao et al., 2016; Sohn et al., 2018). If amyloid pathology has a greater effect on women compared to men, then both $\mathrm{AD}$ and AMD would be exacerbated in women.

The number of homozygous carriers of CFH and ARMS2 SNPs was more than double in the AMD group compared to the control group. This is expected as presence of the CFH and ARMS2 SNPS are said to account for over $50 \%$ of AMD cases (Haines et al., 2005; Haines et al., 2006). Although not statistically significant, homozygosity of the FADS1 SNP was more frequent in the AMD group compared to controls. This agrees with numerous studies reporting the contributions of these SNPs to AMD (Edwards et al., 2005; Hageman et al., 2005; Jakobsdottir et al., 2005; Klein et al., 2005; Haines et al., 2006; Fritsche et al., 2008; Neale et al., 2010). However, FADS1 rs174547 was present in the majority of the sample. To rule out sampling error, the carrier status of FADS1 rs174547 in the original 107 was analyzed.

In the original sample, 91 individuals (85\%) were found to be carriers and of them, 53.9\% were homozygous. The alleles present at rs174547 are C, the ancestral allele, or $\mathrm{T}$. The $\mathrm{T}$ allele is considered the risk allele for AMD. The major allele at this location differs depending on the population. The population of the current study, consisting mostly of individuals of French-Canadian heritage, could be considered most similar to a European or an American population. According to the dbSNP, a database of genetic and epidemiological information on SNPs from the National Institute of Health, the frequency for the $\mathrm{T}$ allele of rs174547 in an American population (0.41) is lower than that of a European population, at 0.65 (NIH, 2017). The frequency of the $\mathrm{T}$ allele in the current study is 0.85 , greater than either the European or American frequencies. One explanation of this could be the Quebec Founder population effect (RoyGagnon et al., 2011). A Founder population is a new population that is established from few individuals (or founders) and, as a result, exhibits reduced genetic variation. Due to this, rare disease alleles are enriched, leading to higher numbers of homozygotes displaying the disease phenotype (Kristiansson et al., 2008). Such populations have been instrumental in medical genetics for research on genetic diseases. The Quebec population has been valuable in the study of genotype-phenotype interactions in Usher syndrome (Ebermann et al., 2009), and retinitis pigmentosa (Koenekoop et al., 2003; Coussa et al., 2015). The Founder Effect could potentially explain the increased frequency of the T allele at rs174547 in this study population.

In addition to occurring with greater frequency, the FADS1 SNP was carried by all participants scoring positive for MCI on the MoCA. Homozygotes had the lowest cognitive scores, suggesting the FADS1 SNP has a greater contribution to cognition than vision. The CFHY402H and ARMS2A69S SNPs appeared not to have an association with the results of cognitive questionnaires, as MoCA scores were not significantly different between carriers and non-carriers. This finding supports the biochemical research on FADS1 discussed in the introduction. The presence of the rs174547 SNP increases delta-5 desaturase activity which, in turn, reduces DHA (Cakiner-Egilmez, 2008; Fauser et al., 2011; Merino et al., 2011; Hellstrand et al., 2012), a vital component for brain structure and cognition.

The Salisbury Eye Evaluation Study (Zheng et al., 2018) established a correlation between deterioration of vision and cognitive decline over an 8-year period. They also determined that the vision problems preceded cognitive decline, however, a causal relationship was not defined. The correlational relationship led to the sensory deprivation hypothesis, which states that a prolonged decline in sensory input will lead to cognitive decline due to neuronal atrophy (Valentijn et al., 2005; Clay et al., 2009). However, there is evidence to show to that vision impairment does lead to reorganization in the brain (Chen et al., 2019), but this is with respect to the processing of visual information, not memory and/or cognition. The few studies that have explored this hypothesis have not found results to support it (Hall et al., 2005; Anstey et al., 2006). Individuals with vision impairment often suffer from social isolation and depression 
(Renaud and Bédard, 2013; Zheng et al., 2018), both of which are also associated with cognitive decline (Potter and Steffens, 2007; Morimoto et al., 2015; Mick et al., 2017). It is important to consider that the relationship between vision and cognitive health could be mediated by these factors. Presently, there has been more evidence to support the common cause hypothesis, which considers aging to affect the physiology of the brain, causing decline in sensory and cognitive functions (Lindenberger and Baltes, 1994). The present study lends further support to this hypothesis by highlighting the potential role of the FADS1 SNP in age-related vision and cognitive decline.

This study is not without limitation, the most obvious being sample size. Since there has been little study on these SNPs in relation to cognitive impairment, there is no established minor allele frequency for cognitive impairment cases, which is required to determine appropriate sample size. Further, the current dataset is non-normally distributed - which means that a sensitivity analysis using $\mathrm{g}^{*}$ power is also not possible. It is hoped the results presented here will be the impetus to support larger scale studies in the future.

\section{CONCLUSION}

Although the prevalence of MCI among participants with AMD was not much higher than controls in this sample, the prevalence is higher than that reported in other normallysighted populations (Gauthier et al., 2006). Additionally, those with AMD scoring positive for MCI according to the MoCA had difficulty with different cognitive domains compared to controls scoring positive for MCI. This distribution of cognitive impairment indicates that those with AMD and MCI may be more likely to progress to AD than controls with MCI.

No significant associations between the most prominent AMD SNPs, CFHY402H and ARMS2A69S, and MCI were identified. This gives support to previous claims that although AMD and $\mathrm{AD}$ have many similarities, the underlying genetic mechanisms may be different (Proitsi et al., 2012). However, the findings were different for the FADS1 SNP. Carriers, both with and without AMD, were more likely to have lower cognitive scores compared to non-carriers. Further, all those scoring positive for MCI according to the MoCA were homozygous for the FADS1 SNP. These findings highlight the importance of testing for not only the prominent AMD SNPs but also the FADS1 mutation in future studies.

Genetic studies of complex disease have recently become possible, but they have required vast study cohorts for an individual trait and international collaborations on enormous scales (Consortium, 2007). Large global populations may not always be necessary to study the genetics of complex diseases, like AMD and cognitive impairment. Susceptibility to complex disease involves contributions from common variants and rare variants. Several common variants are likely to explain a substantial fraction of the genetic contribution to a complex disease, while more rare variants have a greater impact on the phenotype of the disease. The statistical power required to detect susceptibility alleles is positively correlated with the frequency of the allele and the penetrance, or degree of phenotypic expression of the allele in the test population. Founder populations may be required to better define a risk allele, like FADS1 rs174547 that, although significant, gets lost in GWAS as a result of population-specific effects. A number of researchers have discussed the advantages of using Founder populations in medical genetics. Some of the benefits include genetic, environmental and phenotypic homogeneity, good genealogical records, higher degree of linkage disequilibrium, and reduced allelic heterogeneity (Lohmueller et al., 2003; Cohen et al., 2004; Zeggini et al., 2005; Kristiansson et al., 2008). The results of this study suggest FADS1 rs174547 may be a new focus for better understanding any common genetic mechanism in the AMD-MCI co-morbidity.

\section{DATA AVAILABILITY STATEMENT}

The datasets generated for this study can be found in online repositories. The names of the repository/repositories and accession number(s) can be found below: https://osf.io/zt26y/ ?view_only=4993bebe8e20457680fa72f5ee49934c, zt26y.

\section{ETHICS STATEMENT}

The studies involving human participants were reviewed and approved by the Le Comité d'Éthique de la Recherche en Santé at the Université de Montréal. The patients/participants provided their written informed consent to participate in this study.

\section{AUTHOR CONTRIBUTIONS}

All authors contributed to study design, manuscript revision and have read and approved the final submitted version.

\section{FUNDING}

This work was supported by the Common Infrastructures program of the Vision Health Research Network (VHRN) of the Fonds de Recherche du Québec en Santé (FRQS) and doctoral fellowships: The Canadian National Institute for the Blind Ross C. Purse Doctoral Fellowship, the FRQS Doctoral Fellowship and the VHRN/FRQS Bourse de Doctorat en Sciences de la Vision.

\section{ACKNOWLEDGMENTS}

We would like to thank Anneke de Hollander, Ph.D. for her role in the original genotyping and David Nguyen-Tri, Ph.D. for technical support.

\section{SUPPLEMENTARY MATERIAL}

The Supplementary Material for this article can be found online at: https://www.frontiersin.org/articles/10.3389/fnagi.2020. 586691/full\#supplementary-material 


\section{REFERENCES}

Alzheimer, A., Stelzmann, R. A., Schnitzlein, H. N., and Murtagh, F. R. (1995). An english translation of Alzheimer's 1907 paper, "Uber eine eigenartige Erkankung der Hirnrinde". Clin. Anat. 8, 429-431. doi: 10.1002/ca.98008 0612

Anderson, D. H., Talaga, K. C., Rivest, A. J., Barron, E., Hageman, G. S., and Johnson, L. V. (2004). Characterization of beta amyloid assemblies in drusen: the deposits associated with aging and age-related macular degeneration. Exp. Eye Res. 78, 243-256.

Anstey, K. J., Lord, S. R., Hennessy, M., Mitchell, P., Mill, K., and von Sanden, C. (2006). The effect of cataract surgery on neuropsychological test performance: a randomized controlled trial. J. Int. Neuropsychol. Soc. 12, 632-639. doi: $10.1017 / \mathrm{s} 1355617706060954$

Areds Research Group. (2006). AREDS report No. 16: cognitive impairment in the age-related eye disease study. Arch. Ophthalmol. 124, 537-543. doi: 10.1001/ archopht.124.4.537

Augood, C., Chakravarthy, U., Young, I., Vioque, J., de Jong, P. T., Bentham, G., et al. (2008). Oily fish consumption, dietary docosahexaenoic acid and eicosapentaenoic acid intakes, and associations with neovascular age-related macular degeneration. Am. J. Clin. Nutr. 88, 398-406. doi: 10.1093/ajcn/88. 2.398

Baker, M. L., Wang, J. J., Rogers, S., Klein, R., Kuller, L. H., Larsen, E. K., et al. (2009). Early age-related macular degeneration, cognitive function, and dementia: the Cardiovascular Health Study. Arch. Ophthalmol. 127, 667-673. doi: 10.1001/archophthalmol.2009.30

Busse, A., Sonntag, A., Bischkopf, J., Matschinger, H., and Angermeyer, M. C. (2002). Adaptation of dementia screening for vision-impaired older persons: administration of the Mini-Mental State Examination (MMSE). J. Clin. Epidemiol. 55, 909-915. doi: 10.1016/s0895-4356(02)00449-3

Cakiner-Egilmez, T. (2008). Omega 3 fatty acids and the eye. Insight 33, 20-27.

Chen, N., Shin, K., Millin, R., Song, Y., Kwon, M. Y., and Tjan, B. S. (2019). Cortical reorganization of peripheral vision induced by simulated central vision loss. J. Neurosci. 39, 3529-3536.

Chung, S.-D., Lee, C.-Z., Kao, L.-T., Lin, H.-C., Tsai, M.-C., and Sheu, J.-J. (2015). Association between neovascular age-related macular degeneration and dementia: a population-based case-control study in Taiwan. PLoS One 10:e0120003. doi: 10.1371/journal.pone.0120003

Clay, O. J., Edwards, J. D., Ross, L. A., Okonkwo, O., Wadley, V. G., Roth, D. L., et al. (2009). Related decline in memory span and fluid intelligence. J. Aging Heal. 21, 547-566. doi: 10.1177/0898264309333326

Cohen, J. C., Kiss, R. S., Pertsemlidis, A., Marcel, Y. L., McPherson, R., and Hobbs, H. H. (2004). Multiple rare alleles contribute to low plasma levels of HDL cholesterol. Science. 305, 869-872. doi: 10.1126/science.109 9870

Consortium, (2007). Genome-wide association study of 14,000 cases of seven common diseases and 3,000 shared controls. Nature 447, 661-683. doi: 10.1038/ nature 05911

Coussa, R. G., Chakarova, C., Ajlan, R., Taha, M., Kavalec, C., Gomolin, J., et al. (2015). Genotype and phenotype studies in autosomal dominant retinitis pigmentosa (adRP) of the french canadian founder population. Investig. Ophthalmol. Vis. Sci. 56, 8297-8305. doi: 10.1167/iovs.1517104

Craig, J. (2008). Complex diseases: research and applications. Nat. Educ. 1:184.

Dag, E., Örnek, N., Örnek, K., and Türkel, Y. (2014). Mini mental state exam versus Montreal cognitive assessment in patients with age-related macular. Eur. Rev. Med. Pharmacol. Sci. 18, 3025-3028.

Demirci, S., Güneş, A., Demirci, K., Demirci, S., Tök, L., and Tök, Ö (2015). Is Alzheimer disease related to age-related macular degeneration? Turkish J. Med. Sci. 45, 1115-1121.

Dumont, J., Huybrechts, I., Spinneker, A., Grammatikaki, E., Bevilacqua, N., Vyncke, K., et al. (2011). FADS1 genetic variability interacts with dietary $\alpha$-linolenic acid intake to affect serum non-HDL-cholesterol concentrations in european adolescents. J. Nutr. Biochem. Mol. Genet. Mech. J. Nutr. 141, 1247-1253. doi: 10.3945/jn.111.140392

Duponsel, N., Wittich, W., Dubuc, S., and Overbury, O. (2010). Correlation of vision loss and mild cognitive impairment as found on the montreal cognitive assessment (MoCA) scale. Optom. Vis. Sci. 87:100380.
Ebermann, I., Koenekoop, R. K., Lopez, I., Bou-Khzam, L., Pigeon, R., and Bolz, H. J. (2009). An USH2A founder mutation is the major cause of Usher syndrome type 2 in Canadians of French origin and confirms common roots of Quebecois and Acadians. Eur. J. Hum. Genet. 17143, 80-84. doi: 10.1038/ejhg. 2008.143

Edwards, A. O., Ritter, R. III, Abel, K. J., Manning, A., Panhuysen, C., and Farrer, L. A. (2005). Complement factor $\mathrm{H}$ polymorphism and age-related macular degeneration. Science 308, 421-424. doi: 10.1126/science.1110189

Fauser, S., Smailhodzic, D., Caramoy, A., van de Ven, J. P. H., Kirchhof, B., Hoyng, C. B., et al. (2011). Evaluation of serum lipid concentrations and genetic variants at high-density lipoprotein metabolism loci and TIMP3 in age-related macular degeneration. Invest. Ophthalmol. Vis. Sci. 52, 5525-5528. doi: 10.1167/iovs.106827

Freemantle, E., Lalovic, A., Mechawar, N., and Turecki, G. (2012). Age and Haplotype variations within FADS1 interact and associate with alterations in fatty acid composition in human male cortical brain tissue. PLoS One 7:e42696. doi: 10.1371/journal.pone.0042696

Fritsche, L. G., Loenhardt, T., Janssen, A., Fisher, S. A., Rivera, A., Keilhauer, C. N., et al. (2008). Age-related macular degeneration is associated with an unstable ARMS2 (LOC387715) mRNA. Nat. Genet. 40, 892-896. doi: 10.1038/ng.170

Gatta, L. B., Vitali, M., Zanola, A., Venturelli, E., Fenoglio, C., Galimberti, D., et al. (2008). Polymorphisms in the LOC387715/ARMS2 putative gene and the risk for Alzheimer's disease. Dement. Geriatr. Cogn. Disord. 26, 169-174. doi: 10.1159/000151050

Gauthier, S., Reisberg, B., Zaudig, M., Petersen, R. C., Ritchie, K., Broich, K., et al. (2006). Mild cognitive impairment. Lancet 367, 1263-1270.

Guo, L., Duggan, J., and Cordeiro, M. F. (2010). Alzheimer's disease and retinal neurodegeneration. Curr. Alzheimer Res. 7, 3-14. doi: 10.2174/ 156720510790274491

Hageman, G. S., Anderson, D. H., Johnson, L. V., Hancox, L. S., Taiber, A. J., Hardisty, L. I., et al. (2005). A common haplotype in the complement regulatory gene factor $\mathrm{H}(\mathrm{HF} 1 / \mathrm{CFH})$ predisposes individuals to age-related macular degeneration. Proc. Natl. Acad. Sci. U.S.A. 102, 7227-7232. doi: 10.1073/pnas. 0501536102

Haines, J. L., Hauser, M. A., Schmidt, S., Scott, W. K., Olson, L. M., Gallins, P., et al. (2005). Complement factor $H$ variant increases the risk of agerelated macular degeneration. Science 308, 419-421. doi: 10.1126/science.111 0359

Haines, J. L., Schnetz-Boutaud, N., Schmidt, S., Scott, W. K., Agarwal, A., Postel, E. A., et al. (2006). Functional candidate genes in age-related macular degeneration: significant association with VEGF, VLDLR, and LRP6. Invest. Ophthalmol. Vis. Sci. 47, 329-335. doi: 10.1167/iovs.05-0116

Hall, T. A., McGwin, G., and Owsley, C. (2005). Effect of cataract surgery on cognitive function in older adults. J. Am. Geriatr. Soc. 53, 2140-2144. doi: 10.1111/j.1532-5415.2005.00499.x

Harrabi, H., Kergoat, M.-J., Rousseau, J., Boisjoly, H., Schmaltz, H., Moghadaszadeh, S., et al. (2015). Age-related eye disease and cognitive function. Invest. Ophthalmol. Vis. Sci. 56, 1217-1221.

Hellstrand, S., Sonestedt, E., Ericson, U., Gullberg, B., Wirfalt, E., Hedblad, B., et al. (2012). Intake levels of dietary long-chain PUFAs modify the association between genetic variation in FADS and LDL-C. J. Lipid. Res. 53, 1183-1189. doi: 10.1194/jlr.p023721

Hoops, S., Nazem, S., Siderowf, A. D., Duda, J. E., Xie, S. X., Stern, M. B., et al. (2009). Validity of the MoCA and MMSE in the detection of MCI and dementia in Parkinson disease. Neurology 73, 1738-1745. doi: 10.1212/wnl. 0b013e3181c34b47

IBM Corporation. (2011). IBM SPSS Statistics for OSX. Armonk, NY: IBM Corporation.

Jakobsdottir, J., Conley, Y. P., Weeks, D. E., Mah, T. S., Ferrell, R. E., and Gorin, M. B. (2005). Susceptibility genes for age-related maculopathy on chromosome 10q26. Am. J. Hum. Genet. 77, 389-407. doi: 10.1086/444437

JASP Team T (2017). JASP Version 0.8.1.2. Computer software. Amsterdam: JASP. Jefferis, J. M., Collerton, J., Taylor, J. P., Jagger, C., Kingston, A., Davies, K., et al. (2012). The impact of visual impairment on mini-mental state examination scores in the Newcastle 85+ study. Age Ageing. 41, 565-568. doi: 10.1093/ ageing/afs042

Johnson, L. V., Leitner, W. P., Rivest, A. J., Staples, M. K., Radeke, M. J., and Anderson, D. H. (2002). The Alzheimer's A beta -peptide is deposited at sites 
of complement activation in pathologic deposits associated with aging and agerelated macular degeneration. Proc. Natl. Acad. Sci. U.S.A. 99, 11830-11835. doi: 10.1073/pnas.192203399

Jonna, G., Katz, M. S. J., and Fingerhut, D. E. (2013). Cognitive impairment in AMD. Ophthalmology 120:1106. doi: 10.1016/j.ophtha.2013.01.041

Kaarniranta, K., Salminen, A., Haapasalo, A., Soininen, H., and Hiltunen, M. (2011). Age-related macular degeneration (AMD): Alzheimer's disease in the eye? J. Alzheimers Dis. 24, 615-631.

Katta, S., Kaur, I., and Chakrabarti, S. (2009). The molecular genetic basis of agerelated macular degeneration: an overview. J. Genet. 88, 425-449. doi: 10.1007/ s12041-009-0064-4

Keenan, T. D. L., Goldacre, R., and Goldacre, M. J. (2014). associations between age-related macular degeneration, alzheimer disease, and dementia. JAMA Ophthalmol. 132:63. doi: 10.1001/jamaophthalmol.2013.5696

Klaver, C. C., Ott, A., Hofman, A., Assink, J. J., Breteler, M. M., and de Jong, P. T. (1999). Is age-related maculopathy associated with Alzheimer's Disease? Am. J. Epidemiol. 150, 963-968.

Klein, R., Klein, B. E. K., Knudtson, M. D., Meuer, S. M., Swift, M., and Gangnon, R. E. (2007). Fifteen-year cumulative incidence of age-related macular degeneration. Beaver Dam Eye Study. Ophthalmol. 114, 253-262. doi: 10.1016/j.ophtha.2006.10.040

Klein, R. J., Zeiss, C., Chew, E. Y., Tsai, J. Y., Sackler, R. S., Haynes, C., et al. (2005). Complement factor $\mathrm{H}$ polymorphism in age-related macular degeneration. Science 308, 385-389. doi: 10.1126/science.1109557

Koenekoop, R. K., Loyer, M., Hand, C. K., Al Mahdi, H., Dembinska, O., Beneish, R., et al. (2003). Novel RPGR mutations with distinct retinitis pigmentosa phenotypes in French-Canadian families. Am. J. Ophthalmol. 136, 678-687. doi: 10.1016/s0002-9394(03)00331-3

Kristiansson, K., Naukkarinen, J., and Peltonen, L. (2008). Isolated populations and complex disease gene identification. Genome Biol. 9:109. doi: 10.1186/gb-20089-8-109

Lindenberger, U., and Baltes, P. B. (1994). Sensory functioning and intelligence in old age: A strong connection. Psychol. Aging. 9, 339-355. doi: 10.1037/08827974.9.3.339

Lohmueller, K. E., Pearce, C. L., Pike, M., Lander, E. S., and Hirschhorn, J. N. (2003). Meta-analysis of genetic association studies supports a contribution of common variants to susceptibility to common disease. Nat. Genet. 33, 177-182. doi: $10.1038 /$ ng 1071

Lopez, O. L., Jagust, W. J., DeKosky, S. T., Becker, J. T., Fitzpatrick, A., Dulberg, C., et al. (2003). Prevalence and classification of mild cognitive impairment in the Cardiovascular Health Study Cognition Study: part 1. Arch. Neurol. 60, 1385-1389. doi: 10.1001/archneur.60.10.1385

Martinelli, N., Girelli, D., Malerba, G., Guarini, P., Illig, T., Trabetti, E., et al. (2008). FADS genotypes and desaturase activity estimated by the ratio of arachidonic acid to linoleic acid are associated with inflammation and coronary artery disease. Am. J. Clin. Nutr. 88, 941-949. doi: 10.1093/ajcn/88.4.941

Merino, D. M., Johnston, H., Clarke, S., Roke, K., Nielsen, D., Badawi, A., et al. (2011). Polymorphisms in FADS1 and FADS2 alter desaturase activity in young Caucasian and Asian adults. Mol. Genet. Metab. 103, 171-178. doi: 10.1016/j. ymgme.2011.02.012

Mick, P., Pichora-Fuller, M., and Wittich, W. (2017). Associations between sensory loss and social networks, participation, support, and loneliness. Innov. Aging 1(Suppl._1), 732-732. doi: 10.1093/geroni/igx004.2643

Micklisch, S., Lin, Y., Jacob, S., Karlstetter, M., Dannhausen, K., Dasari, P., et al. (2017). Age-related macular degeneration associated polymorphism rs10490924 in ARMS2 results in deficiency of a complement activator. J. Neuroinflamm. 14:4.

Morimoto, S. S., Kanellopoulos, D., and Alexopoulos, G. S. (2015). Cognitive impairment in depressed older adults. HHS Public Access Author Manuscr. 44, 138-142. doi: 10.3928/00485713-20140306-05

Nasreddine, Z. S., Phillips, N. A., Bédirian, V., Charbonneau, S., Whitehead, V., Collin, I., et al. (2005). The montreal cognitive assessment, MoCA: a brief screening tool for mild cognitive impairment. J. Am. Geriatr. Soc. 53, 695-699. doi: $10.1111 /$ j.1532-5415.2005.53221.x

Neale, B. M., Fagerness, J., Reynolds, R., Sobrin, L., Parker, M., Raychaudhuri, S., et al. (2010). Genome-wide association study of advanced age-related macular degeneration identifies a role of the hepatic lipase gene (LIPC). Proc. Natl. Acad. Sci. U.S.A. 107, 7395-7400. doi: 10.1073/pnas.0912019107
NIH. (2017). dbSNP. Bethesda, ML: NIH.

Ohno-Matsui, K. (2011). Parallel findings in age-related macular degeneration and Alzheimer's disease. Prog. Retin. Eye Res. 30, 217-238.

Orr, S. K., and Bazinet, R. P. (2008). The emerging role of docosahexaenoic acid in neuroinflammation. Curr. Opin. Investig. Drugs. 9, 735-743.

Pham, T. Q., Kifley, A., Mitchell, P., and Wang, J. J. (2006). Relation of agerelated macular degeneration and cognitive impairment in an older population. Gerontology 52, 353-358. doi: 10.1159/000094984

Potter, G. G., and Steffens, D. C. (2007). Contribution of depression to cognitive impairment and dementia in older adults. Neurologist 13, 105-117. doi: 10. 1097/01.nrl.0000252947.15389.a9

Proitsi, P., Lupton, M. K., Dudbridge, F., Tsolaki, M., Hamilton, G., Daniilidou, M., et al. (2012). Alzheimer's disease and age-related macular degeneration have different genetic models for complement gene variation. Neurobiol. Aging 33:1843.e9-17.

Renaud, J., and Bédard, E. (2013). Depression in the elderly with visual impairment and its association with quality of life. Clin. Interv. Aging. 8, 931-943. doi: 10.2147/cia.s27717

Rousselet, G. A., Pernet, C. R., and Wilcox, R. R. (2017). Beyond differences in means: robust graphical methods to compare two groups in neuroscience. Eur. J. Neurosci. 46, 1738-1748. doi: 10.1111/ejn.13610

Roy-Gagnon, M. H., Moreau, C., Bherer, C., St-Onge, P., Sinnett, D., Laprise, C., et al. (2011). Genomic and genealogical investigation of the French Canadian founder population structure. Hum. Genet. 129, 521-531. doi: 10.1007/s00439010-0945-x

Rozzini, L., Chilovi, B. V., Conti, M., Bertoletti, E., Delrio, I., Trabucchi, M., et al. (2007). Conversion of amnestic mild cognitive impairment to dementia of Alzheimer type is independent to memory deterioration. Int. J. Geriatr. Psychiatry. 22, 1217-1222. doi: 10.1002/gps.1816

Rozzini, L., Riva, M., Ghilardi, N., Facchinetti, P., Forbice, E., Semeraro, F., et al. (2014). Cognitive dysfunction and age-related macular degeneration. Am. J. Alzheimers Dis. Other. Demen. 29, 256-262.

Serini, S., Fasano, E., Piccioni, E., Cittadini, A. R., and Calviello, G. (2011). Dietary $\mathrm{n}-3$ polyunsaturated fatty acids and the paradox of their health benefits and potential harmful effects. Chem. Res. Toxicol. 24, 2093-2105. doi: 10.1021/ tx200314p

Smailhodzic, D., Klaver, C. C. W., Klevering, B. J., Boon, C. J. F., Groenewoud, J. M. M., Kirchhof, B., et al. (2012a). Risk alleles in CFH and ARMS2 are independently associated with systemic complement activation in age-related macular degeneration. Ophthalmology 119, 339-346. doi: 10.1016/j.ophtha. 2011.07.056

Smailhodzic, D., Muether, P. S., Chen, J., Kwestro, A., Zhang, A. Y., Omar, A., et al. (2012b). Cumulative effect of risk alleles in CFH, ARMS2, and VEGFA on the response to ranibizumab treatment in age-related macular degeneration. Ophthalmology 119, 2304-2311.

Sohn, D., Shpanskaya, K., Lucas, J. E., Petrella, J. R., Saykin, A. J., Tanzi, R. E., et al. (2018). Sex differences in cognitive decline in subjects with high likelihood of mild cognitive impairment due to Alzheimer's disease. Sci. Rep. 8, 1-9.

Summers, M. J., and Saunders, N. L. J. (2012). Neuropsychological measures predict decline to Alzheimer's dementia from mild cognitive impairment. Neuropsychology. 26, 498-508. doi: 10.1037/a0028576

Terai, K., Iwai, A., Kawabata, S., Tasaki, Y., Watanabe, T., Miyata, K., et al. (2001). beta-amyloid deposits in transgenic mice expressing human beta-amyloid precursor protein have the same characteristics as those in Alzheimer's disease. Neuroscience 104, 299-310. doi: 10.1016/s0306-4522(01)00095-1

Tsai, D.-C., Chen, S.-J., Huang, C.-C., Yuan, M.-K., and Leu, H.-B. (2015). Agerelated macular degeneration and risk of degenerative dementia among the elderly in Taiwan. Ophthalmology 122, 2327-2335. doi: 10.1016/j.ophtha.2015. 07.033

Tuo, J., Ross, R. J., Herzlich, A. A., Shen, D., Ding, X., Zhou, M., et al. (2009). A high omega-3 fatty acid diet reduces retinal lesions in a murine model of macular degeneration. Am. J. Pathol. 175, 799-807. doi: 10.2353/ajpath.2009.090089

Uauy, R., and Dangour, A. D. (2006). Nutrition in brain development and aging: role of essential fatty acids. Nutr. Rev. 64(5 Pt. 2), S24-S33.

Valentijn, S. A. M., Van Boxtel, M. P. J., Van Hooren, S. A. H., Bosma, H., Beckers, H. J. M., Ponds, R. W. H. M., et al. (2005). Change in sensory functioning predicts change in cognitive functioning: results from a 6-year follow-up in the 
maastricht aging study. J. Am. Geriatr. Soc. 53, 374-380. doi: 10.1111/j.15325415.2005.53152.x

Weiser, M. J., Butt, C. M., and Mohajeri, M. H. (2016). Docosahexaenoic acid and cognition throughout the lifespan. Nutrients. 8, 1-40. doi: 10.1007/978-3-31909822-7_1

Whitson, H. E., Ansah, D., Sanders, L. L., Whitaker, D., Potter, G. G., Cousins, S. W., et al. (2011). Comorbid cognitive impairment and functional trajectories in low vision rehabilitation for macular disease. Aging Clin. Exp. Res. 23, 343-350. doi: 10.1007/bf03325233

Whitson, H. E., Ansah, D., Whitaker, D., Potter, G., Cousins, S. W., MacDonald, H., et al. (2010). Prevalence and patterns of comorbid cognitive impairment in low vision rehabilitation for macular disease. Arch. Gerontol. Geriatr. 50, 209-212. doi: 10.1016/j.archger.2009.03.010

Wittich, W., Murphy, C., and Mulrooney, D. (2014). An adapted adult day centre for older adults with sensory impairment. Br. J. Vis. Impair. 32, 249-262. doi: 10.1177/0264619614540162

Wittich, W., Phillips, N., Nasreddine, Z., and Chertkow, H. (2010). Sensitivity and specificity of the montreal cognitive assessment modified for individuals who are visually impaired. J. Vis. Impair. Blind 104, 360-369. doi: 10.1177/ $0145482 \times 1010400606$

Wong, T. Y., Klein, R., Nieto, F. J., Moraes, S. A., Mosley, T. H., Couper, D. J., et al. (2002). Is early age-related maculopathy related to cognitive function? the atherosclerosis risk in communities study. Am. J. Ophthalmol. 134, 828-835. doi: 10.1016/s0002-9394(02)01672-0

Woo, S. J., Park, K. H., Ahn, J., Choe, J. Y., Jeong, H., Han, J. W., et al. (2012). Cognitive impairment in age-related macular degeneration and geographic atrophy. Ophthalmology 119, 2094-2101.

World Health Organization (2012). World Alzheimer Report 2012, A Public Health Priority. Geneva: WHO.
Wortmann, M. (2012). Dementia: a global health priority - highlights from an ADI and World Health Organization report. Alzheimers Res. Ther. 4, 40.

Zeggini, E., Rayner, W., Morris, A. P., Hattersley, A. T., Walker, M., Hitman, G. A., et al. (2005). An evaluation of HapMap sample size and tagging SNP performance in large-scale empirical and simulated data sets. Nat. Genet. 37, 1320-1322. doi: 10.1038/ng1670

Zhao, L., Mao, Z., Woody, S., and Brinton, R. (2016). Sex differences in metabolic aging of the brain: neurobiol aging. Neurobiol. Aging 42, 69-79. doi: 10.1016/j. neurobiolaging.2016.02.011

Zheng, D. D., Swenor, B. K., Christ, S. L., West, S. K., Lam, B. L., and Lee, D. J. (2018). Longitudinal associations between visual impairment and cognitive functioning the salisbury eye evaluation study. JAMA Ophthalmol. 136, 989995. doi: 10.1001/jamaophthalmol.2018.2493

Zhou, L. X., Sun, C. L., Wei, L. J., Gu, Z. M., Lv, L., and Dang, Y. (2016). Lower cognitive function in patients with age-related macular degeneration: a meta-analysis. Clin. Interv. Aging. 21, 215-223. doi: 10.2147/cia. s102213

Conflict of Interest: The authors declare that the research was conducted in the absence of any commercial or financial relationships that could be construed as a potential conflict of interest.

Copyright (C) 2020 Murphy, Johnson, Koenekoop, Seiple and Overbury. This is an open-access article distributed under the terms of the Creative Commons Attribution License (CC BY). The use, distribution or reproduction in other forums is permitted, provided the original author(s) and the copyright owner(s) are credited and that the original publication in this journal is cited, in accordance with accepted academic practice. No use, distribution or reproduction is permitted which does not comply with these terms. 